\title{
Myasthenia gravis: A rare presentation
}

\author{
Sandeep Lahiry', Dipak Bhowmik ${ }^{2}$ \\ Post Graduate Trainee; Department of Pharmacology; Institute of Post Gradute Medical Education \& Research, \\ ${ }^{2}$ Medical Director \& ICU Incharge, Dreamland Nursing Home, Kolkata, West Bengal, India
}

Myasthenia gravis (MG) is a relatively rare autoimmune disorder in which antibodies form against acetylcholine (Ach) nicotinic postsynaptic receptors at the neuromuscular junction of skeletal muscles. The usual initial complaint is a muscle weakness but can also present as respiratory failure, as in our case. MG is treated with medications such as Acetylcholineesterase (AChE) inhibitors or immunosuppressants and, in selected cases, thymectomy. Here, we present a case of 32 year old women with MG, who presented with an unexplained respiratory failure. She was stabilized with mechanical ventilation and subsequently treated Access this article online with immunoglobins and thymectomy.

Key words: Myasthenia gravis, Acetylcholine, Immunoglobulin

\section{INTRODUCTION}

Myasthenia gravis can manifest as respiratory failure, and the diagnosis needs to be considered in cases of unexplained respiratory failure. Work up for other causes can be exhaustive, expensive, and hazardous if a high index of suspicion for neuromuscular causes is not maintained.

This case report highlights the importance of considering neuromuscular disorders in cases of unexplained respiratory failure in an acute setting. In an emergency unit or intensive care unit setting with sedation and paralytic use, a thorough neurological examination, especially of proximal muscles, may be challenging. Our patient's muscle weakness went unnoticed at the time of initial presentation, which was later exacerbated by infection.

\section{CASE HISTORY}

A 32 year old woman presented to the emergency room (ER) with low-grade fever, generalized weakness and difficulty in swallowing food and liquid for four to five weeks. While in the ER, she complained of respiratory trouble. She initially presented at a rural health centre, so she was transferred to our facility for further management.
On admission, she was conscious, alert and co-operative to verbal commands (GCS $=15 / 15)$. Her vital signs were stable though she complained of respiratory trouble. Arterial blood gas analysis revealed compensated metabolic alkalosis. She was lean and thin, with a body weight of $48 \mathrm{~kg}$. General survey was unremarkable. Neck veins were nonengorged. No palpable neck nodes elicited. Oral cavity \& neck examination were within normal limits.

She which had been complaining of tingling sensation in her limbs, increasing over past few weeks. Dysphagia to both solid and liquids were troubling her for over past 6 weeks. Communication was difficult due to mild conductive deafness in her right ear. She was non-diabetic, non-hypertensive and euthyroid.

Chest auscultation indicated bilateral basal soft crackles and normal heart sounds. Chest skiagram revealed increased hyperlucency of basal zones and prominent bronchovascular markings. Abdominal examination was normal. USG (whole abdomen) revealed mild hepatomegaly. ECG \& Echocardiography were also within normal limits. Anterior chest examination was unremarkable.

Her initial neurological examination had no evidence of focal neurological deficit. Bilateral plantar responses were

\section{Address for correspondence:}

Sandeep Lahiry. Post Graduate Trainee; Department of Pharmacology; Institute of Post Gradute Medical Education \& Research, Kolkata, West Bengal, India E-mail: sndplry@gmail.com, Phone: +91-9432879503 (C) Copyright AJMS 
flexor. Pupils were semi-dialated, normally responding to light. Left sided facial deviation indicated a possibility of 7 th nerve palsy. Power in upper limbs (UL) were: proximal: 4/5, distal: 4/5; Lower limbs - proximal: 3/5, distal: 3/5. Deep tendon reflexes were preserved. Non-contrast CT (Brain) and MRI (Brain) imaging revealed no obvious pathology.

Initial laboratory tests revealed elevated white blood cell count $(14,000 / \mathrm{cm} 3)$ with neutrophilic predominance $(80 \%)$ and C-reactive protein 65.4. Complete blood count, electrolyte panel, hepatic \& renal parameters were normal. Otherwise routine urine analysis was within normal limits.

In spite of near normal respiratory parameters, the patient complained of persistent shortness in breath. A spiral computed tomography scan of the chest was taken, to exclude the possibility of pulmonary embolism or mediastinal lesions like thymoma. There was no evidence of pulmonary embolism on the scan. Moderate hyperplasia of Thymus gland was noted.

An MRI screening (High Cervical Spine) later revealed partially desiccated C2-3, C3-4 \& C5-6 intervertebral disks. A mild posterior disc bulge at C6-7 level was also found to be causing minimal indentation over the anterior thecal sac. No significant spinal stenosis was detected at any level. Cerebrospinal fluid examination was within normal limits. Since most neurodegenerative pathology, space-occupying lesions or intracranial infection was ruled out, MG was suspected.

Within the next 12 hours she had a sudden episode of desaturation, and started complaining of her throat "closing down". She was immediately intubated and mechanically ventilated, assuming a bulbar paralysis. After the intubation she was hypotensive, needing low dose vasopressors for three to four hours. She was started on intravenous fluids and piperacillin-tazobactam (4.5 g thrice daily). Her haemodynamic measures improved with treatment, and she was afebrile within the next 48hrs. Urine and endotracheal secretion cultures revealed 'no - growth' after 48-hrs incubation'.

On Day 3, arterial blood gases did not show any evidence of an A/a gradient or hypoxia. In spite of such parameters the patient failed repeated attempts at extubation. She had also developed left-sided facial weakness with slurring of speech and paraparesis. This led us to further transpire a neuromuscular condition, and, at this point, a more detailed history was obtained from her and her family. It was found that prior to hospitalisation; she had symptoms of upper and lower extremity weakness for the past two months. She had also had blurred vision, minimal problems with swallowing, sensation of choking on lying down, and feeling of being excessively tired by the end day.

A second neurological examination revealed bilateral weakness of the muscles like orbicularis oculi, deltoids, biceps, triceps, interossei, ankle dorsiflexors, and plantar flexors. Rest of the neurological examination including sensory examination was normal. Her weaning parameters were: vital capacity $500 \mathrm{ml}$, negative inspiratory force (NIF) $228 \mathrm{~cm} \mathrm{H} 2 \mathrm{O}$ (normal $235 \mathrm{~cm} \mathrm{H} 2 \mathrm{O}$ or less), and tidal volume $345 \mathrm{ml}$. The rapid shallow breathing index was 75. These findings suggested respiratory muscle weakness. Neuro-physiological studies and ACh receptor antibodies were requested for confirmation. Nerve conduction (NCV) and Electromyography (EMG) studies showed significant decrement on repetitive nerve stimulation (RNST), consistent with the diagnosis of MG.

The patient was started on intravenous Immunoglobulin (0.4 U/kg body wt.) and thus continued for next 5-6 days. Low dose steroid and pyridoxine was also added to therapy. Within the first 48 hrs of immunoglobulin therapy, her vital capacity improved from $500 \mathrm{ml}$ to $1.4 \mathrm{l}$ and the NIF increased to $280 \mathrm{~cm} \mathrm{H} 2 \mathrm{O}$. The next day she passed the weaning trial and was successfully extubated. On completion of 5-day course, there was continued improvement of muscle strength during the rest of her stay in hospital. The ACh receptor antibody panel was positive for binding, blocking, and modulating antibody. She was discharged after two weeks on steroid taper, azathioprine, and pyridostigmine.

At a later date she underwent a thymectomy and had an uneventful postoperative course.

\section{DISCUSSION}

MG has a prevalence of $50-125$ cases per million population. ${ }^{1}$ Respiratory muscle failure is not uncommon in this disorder. Individuals of all ages are affected, however, young women are predisposed with a peak incidence in the twenties. ${ }^{2}$ Clinical features include fatigue and muscle weakness, but reflexes are usually retained. Weakness increases with sustained muscle activity and is attenuated by rest and sleep. Cranial muscles are involved early, diplopia and ptosis as presentation. Dysphagia can also be a feature as seen in our patient.

Generalized weakness occurs in $85 \%$ of patients. $^{2}$ Muscle weakness tends to be more when the muscles are stressed, though strength improves with rest. Preserved 
sensation and reflexes are distinguishing factors from other neuropathies. Vital capacity, timed forward abduction, and muscle dynamometry can be used as objective tools to diagnose and assess the disease activity.

Respiratory muscle involvement in MG can be of two types: myasthenic and cholinergic crises. The term 'myasthenic crisis' is used to describe muscle weakness due to reduced neuromuscular transmission at the synapse. In contrast, 'cholinergic crises' occur because of excessive depolarisation at the neuromuscular junction. Myasthenic crisis can occur as a result of infection, as in our patient, decreased anticholinergic medication, use of aminoglycoside antibiotics, and postoperative stress etc. There are recent reports of fluoroquinolones exacerbating myasthenic weakness. ${ }^{3}$

The normal A/a gradient and clinical features out of proportion with the pulmonary pathology led us to look for other causes of respiratory failure. It has been described that early detection of respiratory muscle involvement can be difficult because of a normal breathing pattern and sometime selective affection of the diaphragmatic and intercostal muscles. ${ }^{4}$

The basic pathophysiology involves a decrease in the number of acetylcholine (ACh) receptors at neuromuscular junctions where vesicles containing ACh are released. Acetylcholinesterase (AChE) in the clefts rapidly hydrolyses the ACh and terminates its action on the muscle. Drugs such as edrophonium act by increasing the concentration of $\mathrm{ACh}$ at the neuromuscular junction. It is now accepted that such changes are due to an 'antibody-mediated process'. Diagnostic tests are needed to confirm clinical suspicion include AChE test, Repetitive Nerve Stimulation Test (RNST), and ACh receptor antibody test. Edrophonium is the drug of choice for the AChE test because of its rapid onset and short half-life (few minutes). The test is considered positive if there is considerable improvement in muscle strength. In the RNST, electricity is delivered to the nerve, and action potentials are recorded on the surface of the muscle. The test is considered positive if there is decremental response of $15 \% .{ }^{5} \mathrm{ACh}$ receptor antibody testing is done with radioimmunoassay (RIA) and is positive in $85 \%$ of patients with generalized MG.

Associated conditions, including thymic tumours and thyroid dysfunction (both hypothyroidism and hyperthyroidism) should be considered since both can exacerbate MG. ${ }^{6}$ Other possibilities are Lambert-Eaton syndrome, drug induced myasthenia, botulinism, and intracranial lesions.

Current treatment options for MG include AChE agents, surgical thymectomy, immunosuppression, and short term but fast acting therapies such as plasma exchange and intravenous immunoglobulin. In general $\mathrm{AChE}$ agents are used as the first line agents along with surgical thymectomy or immunosuppression.

\section{Key messages}

This case report highlights the importance of considering neuromuscular disorders like MG in cases of unexplained respiratory failure in an acute setting. In an ER/intensive care unit setting with sedation and paralytic use, a thorough neurological examination, especially of proximal muscles, may be challenging.

\section{REFERENCES}

1. Kurtzke JF and Kurland LT. The epidemiology of neurological disease. In: Joynt. RJ, eds. Clinical Neurology. Revised edn, Philadelphia: JB Lippincott, 1992; 80-88.

2. Drachman DB. Medical progress: myasthenia gravis. $\mathrm{N}$ Engl $\mathrm{J}$ Med. 1994; 330: 1797-1810.

3. Tintinalli J. Fluoroquinolones should be avoided in myasthenia gravis. Ann Emerg Med 2004; 44:87-88.

4. Gracey DR, Divertie MB and Howard FM. Mechanical ventilation for respiratory failure in myasthenia gravis. Mayo Clin Proc 1983; 58:597-602.

5. Keesey JC. Electrodiagnostic approach to defects of neuromuscular transmission. Muscle Nerve 1989; 12:613-626.

6. Ferguson IT, Murphy RP and Lascelles RG. Ventilatory failure in myasthenia gravis. J Neurol Neurosurg. Psychiatry 1982; 45: $217-222$

Authors Contribution:

SL - Reviewed the literature, manuscript preparation and critical revision of the manuscript; DB - Concept and design of the study, collected data and review of study.

Source of Support: Nil, Conflict of Interest: None declared. 